\title{
LOCAL TOPOLOGICAL MODIFICATION OF HEXAHEDRAL MESHES PART I: A SET OF DUAL-BASED OPERATIONS
}

\author{
Timothy J. Tautges ${ }^{1}$, Sarah E. Knoop ${ }^{2}$, and Thomas J. Rickmeyer ${ }^{3}$
}

Abstract. For hexahedral meshes, it is difficult to make topological modifications which preserve conformal property and which are local to the modified elements. This is most easily understood in how changes affect the dual surfaces ("sheets") and lines ("chords"), which have non-local extent in hexahedral meshes. A set of three operations is proposed which represent distinct local changes to the hex mesh and its dual. These operations are shown to compose the larger set of "flipping" operations described by Bern et. al. The relation between these dual-based operations and the insertion of a Boy surface in the dual, described by Bern, is also discussed.

\section{INTRODUCTION}

Finite element analysis and other methods for numerical solution of PDE's require the decomposition of space into a "mesh" (a polyhedral complex where each polyhedron has a prescribed topology). Such decompositions are often required to be conformal [1], where elements fill space, constitute a topology, and whose intersections are either empty or consist of lowerdimensional elements of the topology. Two types of elements are most common in FEA, hexahedra and tetrahedra. Hexahedral meshes are preferred for some kinds of analysis because of their accuracy for a given amount of compute cost [2][3] ${ }^{4}$ A general-purpose method for generating allhexahedral meshes of suitable geometric quality for FEA has not yet been demonstrated. We assert that an important part of any reliable method will be the ability to locally modify the connectivity of the hex mesh; we refer to this as "local hex mesh modification". Previous efforts to describe hex mesh modification have been either ad-hoc or have not described the relation to the hex mesh dual. In this paper, a set of dual-based hex mesh modification operations are described. We demonstrate how other known modifications can be composed from our set of operations. The paper concludes with a discussion of the Boy surface and how it relates to these operations.

Body-fitted hexahedral mesh generation has been a topic of study for over ten years. Algorithms can be loosely classified into two groups. "Inside-out" algorithms start by filling a solid with a prescribed regular mesh, usually cartesian, then trim the mesh to match the boundary geometry and topology [4][5]. These meshes often have poor quality near the boundary which, for some applications, is precisely where good quality is most needed. These algorithms also tend to produce more hex elements than is strictly necessary. Inside-out algorithms are not widely used for these reasons. "Outside-in" or advancing-front algorithms start with a prescribed quadrilateral boundary mesh, and seek to fill the interior with hex elements. Methods have been investigated for doing this geometrically [6] or based on connectivity [7], with some of the connectivity-based methods making some boundary modifications [8] or losing some advancing front qualities [9][10] in order to improve chances of closing the mesh. Where mesh closure was reliably achieved [9], mesh quality typically suffers; in fact, virtually all these methods left hexahedral meshes whose geometric embedding was of insufficient quality to support FEA.

Local modification of mesh connectivity is a crucial part of virtually all "free-meshing" algorithms. For example, the well-known Delaunay algorithm for generating tetrahedral meshes,

\footnotetext{
1 Argonne National Laboratory, Mathematics and Computer Science Division, Madison, U.S.A.; e-mail: tautges@mcs.anl.gov

2 University of Wisconsin-Madison, Madison, U.S.A.

3 University of Wisconsin-Madison, Madison, U.S.A.; e-mail: tom.rickmeyer@gmail.com

4 Although this is accepted as conventional wisdom in some circles, there is virtually no publication record to support this view. It would be quite helpful if there was a rigorous explanation for why hex meshes perform better than tetrahedral meshes in specific cases.
} 
when applied in three dimensions, leaves sliver or flat elements, which are typically removed using local modifications [11]. All-quadrilateral mesh generation uses similar techniques [12][13]. These operations also play an important role in adaptive mesh refinement on tetrahedral meshes [14]. It is expected that local modification of hex mesh connectivity will be part of any successful hex meshing algorithm. The purpose of this research is to develop such methods using dual-based operations.

\section{2 . PREVIOUS WORK}

There have been only a few previous efforts at local hex mesh modification. Knupp \& Mitchell studied local modifications in the context of mesh optimization. They described "pillow", "inflate hex ring", and "collapse hexes" operations[15]. Some of these operations were also observed in previous work on whisker weaving [7][16]. Although having some overlap with the work described here, this work did not attempt to organize the modification operations systematically or show that other modifications could be composed from them. As an aside, it should be noted that this work did show examples of hex meshes which had poor-quality regions on the interior of the mesh, and which could not be improved by optimizing vertex positions. By this, we conclude that topological modifications in those regions are necessary to obtain good mesh quality, and also that these are not geometric affects attributable to the geometric boundary.

The most systematic work on local hex mesh modification is that of Bern et. al [17]. In this work, a set of six operations were described based on lifting into four dimensions and flipping between two mesh states for each operation. These six operations are shown in Figure 1. This operation set has the satisfying aspect of being "complete" by some measure, namely those operations which can be derived from lifting and flipping. However, Bern et. al also describe a mesh modification which cannot be composed from their six operations: a modification which results in the insertion of a Boy surface into the hex mesh dual. They show how this operation changes the parity of a hex mesh (i.e. the odd/even number of hexes). None of the other six operations changes mesh parity.

In this paper, a smaller set of three dual-based operations is described. This operation set forms a basis for Bern's operations; that is, Bern's operations can be composed from the dual-based operations, and none of the dual-based operations can be reproduced as a combination of others in our set.

\subsection{Nomenclature}

In this paper we discuss only all-hexahedral meshes. In other work, these have been referred to as a "cubification" [18] or "cuboid" mesh [17].

We restrict our discussion here to meshes of maximal dimension 3. Under this assumption, a mesh is a set of entities $\mathrm{M}_{\mathrm{i}}, \mathrm{d}=1 . .3$, containing a topology $\mathrm{T}$. The dual of the mesh is a bijective mapping $\mathrm{T} \rightarrow \mathrm{T}^{\prime}$, mapping each $\mathrm{M}_{\mathrm{i}}^{\mathrm{d}}$ to $\mathrm{D}^{3-\mathrm{d}}$, under the same topology. This definition holds for both hexahedral and tetrahedral meshes, where the dual of a tetrahedral mesh is the well-known Voronoi diagram. The hexahedral mesh dual differs from that of a tetrahedral mesh by having additional non-local structure. Specifically, for a hex mesh dual, the 2-cells $\mathrm{D}_{\mathrm{i}}$ corresponding to each principal direction in a hex can be combined into "sheets" $S_{j}$, which also extend to neighboring hexes through those same edges. Likewise, 1-cells combine into "chords" $\mathrm{C}_{\mathrm{k}}$, which extend to neighboring hexes through the primal faces.

We further restrict ourselves to considering only "conformal" meshes, which, in both the dual and the primal, form cell complexes. That is, in both the dual and the primal, any two d-cells are either disjoint or share a d'-cell, $\mathrm{d}^{\prime}<\mathrm{d}$. In a cell complex, all cells must be simply connected.

The dual also forms a simple arrangement of surfaces [19], where (in 3 dimensions) the surfaces intersect only pairwise to form lines (chords) and in triples to form dual vertices. Qualitatively, the 
constraints that sheets and chords have non-local extent in the mesh and must intersect to form a simple arrangement is an indication of the difficulty of deriving a hex mesh starting from a prescribed boundary of quadrilaterals.

In addition to viewing the mesh in both the primal and dual spaces, we also represent the mesh as a series of sheet diagrams (see Figure 2). The sheet diagram shows the intersections of a sheet with other sheets, and is drawn as a set of vertices, edges and 2-cells. If the dual sheet intersects the outer boundary of the primal mesh, the sheet diagram is drawn with a solid line as its outer boundary, where each vertex on that line is dual to a boundary quadrilateral in the primal mesh. Sheet diagrams are useful for indicating changes to hex mesh topology, and are used extensively in this paper.

\section{3 . DUAL-BASED HEX MESH MODIFICATION OPERATIONS}

Certain local modifications to hex meshes were described by Mitchell [15] and Bern [17], with some operations described in both these works. When viewed in the primal, these operations appear distinct and unrelated. However, when viewed in the dual, it becomes apparent that there are more fundamental operations being performed on dual sheets and chords. In fact, we identify three dual-based operations which are atomic (i.e. each cannot be reproduced as a linear combination of the other two) and which can be used to compose Bern's flips.

\subsection{Three Dual-Based Operations}

The three dual-based operations are named the Atomic Pillow (AP), Open-Collapse (OC) and Face Shrink (FS).

The Atomic Pillow is so-named to distinguish it from the pillow operation described in other work [16]. The Atomic Pillow operation, shown graphically in Figure 3, inserts a new dual surface such that it intersects a dual 1-cell. In the primal, the operation can be viewed as "pulling apart" a quadrilateral into two quads and filling the created void space with two hexahedra sharing five new quadrilateral faces. By itself, this operation always results in a mesh which most applications would consider ill-formed. However, when combined with other operations and with this same operation applied to other faces in the mesh the result can be a well-formed mesh (for example, see the following section, where the composition of Bern's $(3,0)-(0,3)$ is described).

One version of the Open-Collapse operation is shown in Figure 4. As the name implies, this operation has two parts. First, several primal entities are duplicated and "pulled apart" (e.g. edge $a b$ and faces $a f e b$ and abgh in Figure 4), creating a void in the mesh bounded by the original and duplicate entities. In the second part of the operation, the void is collapsed by merging entities pairwise, with both entities in a given pair coming from either the original or the duplicated entities from the first step (in Figure 4 vertices $f$ to $h$ and $e$ to $g$; edges $a f$ to $a h, f e$ to $h g$, and $e b$ to $g b$ ). The Open-Collapse can be performed in other locations, pulling apart and joining other combinations of facets in the primal, and a more general description of this operation appears later in this paper. The Open-Collapse operation does not change the number of hex elements or quad faces in a mesh, but can change the number of primal vertices and edges.

The Face Shrink, shown in Figure 5, operates on a quadrilateral and the two hexahedra it bounds in the mesh, "shrinking" it by pulling away four new primal vertices and edges. This forms a ring of hexes around the original two hexes (the ones sharing the original quad face). In the dual this operation does not form new sheets, but introduces four new dual vertices (hex elements in the primal) by "pushing" two sheets through each other across a 1-cell (see Figure 5, top).

\subsection{Composing Bern's Flipping Operations}

The set of dual-based operations described above (hereafter referred to as the "dual flips") is a 
smaller set than Bern's flips, containing three operations instead of six. Furthermore, Bern's flipping operations can be expressed as combinations of the dual flips, as follows.

The composition of the $(3,0)-(0,3)$ flip is shown in Figure 6 . Starting with a single hex element, an AP is applied to one of the bounding faces (step a). A FS operation is then applied to the face shared by the original hex and one of the hexes resulting from the AP. The AP and FS operations add two and four hexes, respectively, for a total of six new hex elements. This operation has been referred to as a "pillow" operation [16], where a new dual surface is inserted such that it surrounds one or more dual vertices in a mesh. We refer to that operation as a "traditional pillow".

The $(2,0)-(0,2)$ flip is shown in terms of sheet diagrams in Figure 7 . The remaining operations in Bern's flips are reconstructed from dual flips as shown in Figure 8-Figure 11.

\section{4 . DISCUSSION}

A number of important questions arise in the formulation of the set of 3 dual-based operations; these issues are discussed below.

\subsection{Is AP really atomic?}

Recall that the AP is the insertion of a new dual surface into the dual arrangement such that it intersects an existing 1-cell (and, thus, four 2-cells and 3-cells). Also, the "traditional" pillow (i.e. the $(3,0)-(0,3)$ flip) appears similar to the AP, but with the new dual surface intersecting a larger neighborhood of the arrangement (in fact, "surrounding" a 0-cell in the arrangement). The question arises: can an inserted dual surface intersect less of the 3D arrangement, providing an operation which can compose what we call an AP? Consider the case of the dual surface intersecting a 2-cell but not its bounding 1-cells (see Figure 12). Right away, we see that the result is not a valid cell complex, since it contains a non-simply-connected 2-cell. The primal for this arrangement contains a "dangling" quadrilateral in the mesh, bounded by the edge corresponding to the intersected 2-cell in the dual, but also containing 3 other dangling edges (i.e. edges bounding only that quad) and two dangling nodes. Clearly, this mesh is not conformal, violating one of the conditions required for the mesh to be valid.

If the inserted dual surface intersects the arrangement only in a 3-cell, a similar result occurs, this time as a dangling mesh edge and vertex on the interior of the mesh (see Figure 12, bottom right). Again, this forms a non-conformal mesh.

From this, it is concluded that yes, the AP operation is atomic, or at least cannot be composed by intersecting the new dual surface with a smaller neighborhood of the arrangement.

\subsection{Can FS be described as a combination of AP and OC operations?}

Consider performing the AP and OC operation on two quadrilateral faces bounding two hexes, as shown in Figure 13, a). We refer to this composition as a "chord push", since it looks like pushing two chords through each other on the sheet diagrams. Repeat the chord push on two more quad faces, followed by an OC to join the two interior chords into a single chord (Figure 13, b). Perform this composite operation (an AP, OC, and OC) on the two remaining sides of the hex pair (Figure 13, c), concluded by one last OC (which splits the single cyclic chord back into two chords) (Figure 13, d). In the primal, the result is the two original hexes, surrounded by a 4-sided ring of elements, where each segment of the ring consists of two hexes sharing two faces. Now, viewing only the interior half of the ring and the two original hex elements, we see that applying the reverse FS operation brings us to an arrangement resulting from applying the forward operation to the original two hexes (Figure 13, e). If the original arrangement is referred to as $\mathrm{O}$ and the chord push as $\mathrm{CP}$, this operation sequence is:

$$
\mathrm{O}+\mathrm{CP}+\mathrm{CP}+\mathrm{OC}+\mathrm{CP}+\mathrm{OC}+\mathrm{CP}+\mathrm{OC}+\mathrm{OC}-\mathrm{FS}=\mathrm{O}+\mathrm{FS}
$$

This series of operations leads one to believe there is some formal algebra which these 
operations are following. If these operations obeyed associative properties of algebraic arithmetic, one could reduce this to an expression for FS. This topic is beyond the scope of this paper, but would be an interesting one for further study.

\subsection{General OC Operation}

The OC operation as described above, working on two quadrilaterals and their shared edge in the primal, is one of several manifestations of this operation. To see other examples, consider three quadrilaterals sharing a vertex, with one of the quads having had an AP performed on it (Figure 14, top left). Applying the OC as described earlier pulls apart edge $b k$ and quads $b c d k$ and $b k j i$, and joins node $i$ to $c$, and $j$ to $d$ (Figure 14, top right). Next, node $k$ is pulled apart (making a new node $k^{\prime}$, and pulling apart quads $g d k f$ and $k d e f$ and edges $k d$ and $k f$ ) and node $g$ is joined to node $e$ (joining edges and quads having the same topology in the process). The result is the arrangement in Figure 14, bottom right. However, that same arrangement can be achieved in a single step, using the same type of OC operation. Starting at Figure 14, bottom left, pull apart node $k$ (making a new node $k^{\prime}$ and pulling apart quads $b k j i, b c d k, j k f g$ and $k d e f$, and edges in those quads connected to $k$ ), then join nodes $i$ to $c, j$ to $d$, and $g$ to $e$ (and edges and quads having the same topology, as before). The result is the same arrangement on Figure 14, bottom right. From this process emerges a more general statement of the Open/Collapse operation, which can be described as:

- Identify a patch of quadrilaterals on the interior of the mesh which:

- Forms a manifold which is simply connected

- Is split into two groups of quads where each group is simply-connected and combinatorially equivalent to the other (i.e. each entity in one group has a corresponding entity in the other)

- Has a series of edges splitting the two groups of quads

- Split the quads, edges and nodes on the interior of the patch, producing two patches which share their boundary

- Merge each entity in one group to its corresponding entity in the other group

In Figure 14, patches of quadrilaterals participating in each operation are drawn in blue, with the series of edges splitting each patch drawn in red.

Although described here for patches of only two and four quads, this operation should apply to arbitrary patches which satisfy the above requirements. Also, this operation clearly affects the valence of nodes on the patch (including new nodes produced during the operation). We assert that these changes could be directed such that node valence moves toward optimal values (in three dimensions a valence of six is ideal). Finally, we are still investigating how this operation appears in the dual and whether any concepts are easier to understand from that perspective. So far, it seems like viewing this operation on a patch of quadrilaterals is more straightforward.

\subsection{Relation to Boy Surface}

An important question which arises concerning the three dual-based operations is whether they form a complete set for hexahedral mesh modification. That is, can one transition between any two meshes having the same boundary using only these operations? If Bern's operations are complete, then the dual-based operations would be as well. However, Bern pointed out that each of their operations did not change the parity of the mesh; in other words, the number of hexes stayed odd or even through the operation. Furthermore, Bern noted that inserting a Boy surface in the dual of a mesh would change its parity. Schwartz then showed that a mesh containing a Boy surface in its dual was geometrically realizable[20]. Clearly, then, neither set of operations is complete. This subject is addressed in Part II of this paper. 


\section{SUMMARY AND FUTURE WORK}

This paper describes three dual-based operations for making local modifications to a hexahedral mesh. These operations can be used to reproduce six operations described by Bern et. al. The absence of a parity-changing operation indicates that the dual-based set is not complete. However, it also seems likely that the insertion of a Boy surface into an existing dual is related to the atomic pillow operation described here. In particular, we conjecture that an atomic pillow operation can be composed from the insertion of two Boy surfaces and a series of Open/Collapse operations on the results. We also speculate that the Face Shrink operation can be composed in a similar fashion. This is part of the focus of the second part of this paper, and will be the topic of further work.

The Atomic Pillow and Open-Collapse operations, when applied in series in a two-hex arrangement, seem to imply a formal algebra governing these operations. Although outside the scope of this paper, this algebra would be an interesting topic of further study.

Although the operations described here are capable of performing local modifications of a hexahedral mesh, it remains to demonstrate how they can be used to improve the shape of geometric meshes for real examples. This will also be the focus of future work.

\section{6 . ACKNOWLEDGMENTS}

The authors wish to thank the students of the CEMRACS summer school (Katerina Jurkova, Raphael Kuate, Franck Ledoux, Hamdi Zorgati) for their insightful comments and their help getting this information documented correctly.

\section{REFERENCES}

[1] Herbert Edelsbrunner, "Geometry and topology for mesh generation", Cambridge University Press, 2001.

[2] Steven E. Benzley, Ernest Perry, Karl Merkley, Brett Clark, Greg Sjaardema, “A Comparison of All Hexagonal and All Tetrahedral Finite Element Meshes for Elastic and Elasto-plastic Analysis", Proc. 4th Int. Meshing Roundtable, SAND95-2130, Sandia National Laboratories, Albuquerque, New Mexico, September 1995.

[3] Deepa Pakal, Muralidhar Seshadri, Scott A. Canann, Sunil Saigal, "A Comparative Study of Hexahedral and Tetrahedral Elements", report to Sandia National Laboratories, July 1998.

[4] Robert Schneiders, "A Grid-Based Algorithm for the Generation of Hexahedral Element Meshes", Engineering with Computers, 12:168-177 (1996).

[5] R. Taghavi, "Automatic, Parallel and Fault Tolerant Mesh Generation from CAD", Engineering with Computers, 12:178-185 (1996).

[6] Michael B. Stephenson, Scott A. Canann, Ted D. Blacker, "Plastering: A New Approach to Automated, 3D Hexahedral Mesh Generation; Progress Report I", Sandia National Laboratories, SAND89-2192, February 1992.

[7] T. J. Tautges, T. Blacker and S. A. Mitchell, 'The Whisker Weaving Algorithm: A Connectivity-Based Method for Constructing All-Hexahedral Finite Element Meshes', Int. J. Numer. Meth. Eng., 39, 3327-3349 (1996).

[8] M. Muller-Hannemann, "Hexahedral Mesh Generation by Successive Dual Cycle Elimination”, Engineering with Computers 15, pp. 269-279, 1999.

[9] N. T. Folwell and S. A. Mitchell, "Reliable Whisker Weaving via Curve Contraction", Proc. 7th Int. Meshing Roundtable, Sandia National Laboratories, Albuquerque, New Mexico, October 1998.

[10] Franck Ledoux and Jean-Christope Weill, “An Extension of the Whisker Weaving Algorithm", 16 ${ }^{\text {th }}$ International Meshing Roundtable,Seattle, Washington, October 20007.

[11] H. Edelsbrunner, Damrong Guoy, “An Experimental Study of Sliver Exudation”, 
C. Dobrzynski, P. Frey, Ph. Pebay, Editors

Engineering with Computers, 18:3, 2002.

[12] Scott Canann, Sella Muthukrishnan, Bob Phillips, "Topological Improvement Procedures for Quadrilateral and Triangular Finite Element Meshes", Proc. 3rd International Meshing Roundtable, Sandia National Laboratories, 1994.

[13] Paul Kinney, "CleanUp: Improving Quadrilateral Finite Element Meshes", Proc. 6th Int. Meshing Roundtable, SAND97-2399, Sandia National Laboratories, Albuquerque, New Mexico, October 1997.

[14] B. Joe, Construction of Three-Dimensional Improved-Quality Triangulations Using Local Transformations, SIAM J. Sci. Computing, 16:1292-1307, November, 1995.

[15] Patrick Knupp, Scott A. Mitchell, Integration of Mesh Optimization with 3D All-Hex Mesh Generation, Sandia National Laboratories Technical Report SAND99-2852, 1999.

[16] Scott A. Mitchell and Timothy J. Tautges, "Pillowing Doublets: Refining a Mesh to Ensure that Faces Share at Most One Edge", Proc. 4th Int. Meshing Roundtable, SAND95-2130, Sandia National Laboratories, Albuquerque, New Mexico, September 1995.

[17] Bern, D. Eppstein, J. Erickson, "Flipping Cubical Meshes", Engineering with Computers, 18(3):173-187 (2002).

[18] Louis Funar, "Surface quadrangulations mod flips", arXiv:math/0501550v2, http://www.arxiv.org/abs/math/0501550.

[19] Timothy J. Tautges, Sarah E. Knoop, "Topology modification of hexahedral meshes using atomic dual-based operations", Proceedings, 12th International Meshing Roundtable, Sandia National Laboratories report SAND 2003-3030P, pp.415-423, Sept. 2003.

[20] A. Schwartz, "Constructions of Cubical Polytopes", Phd Dissertation, TU Berlin, 2003. Available at http://edocs.tu-berlin.de/diss/2004/schwartz alexander.html. 


\section{ESAIM: PROCEEDINGS}

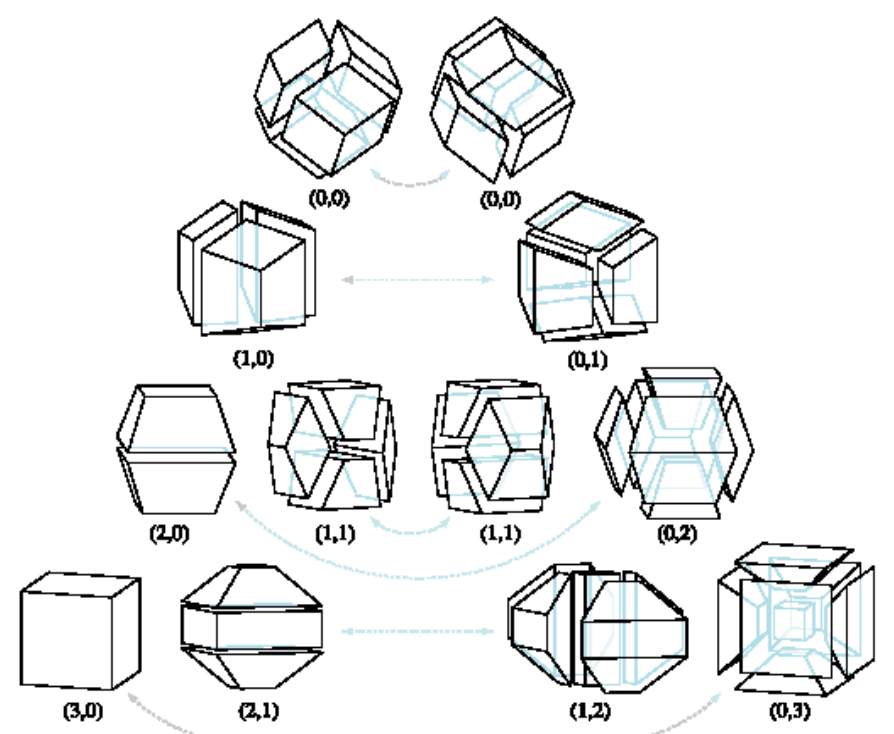

Figure 1: Bern's operations, from Ref. [1]. 
ESAIM: PROCEEDINGS, August 2008, Vol. 24, p. 14-33

C. Dobrzynski, P. Frey, Ph. Pebay, Editors
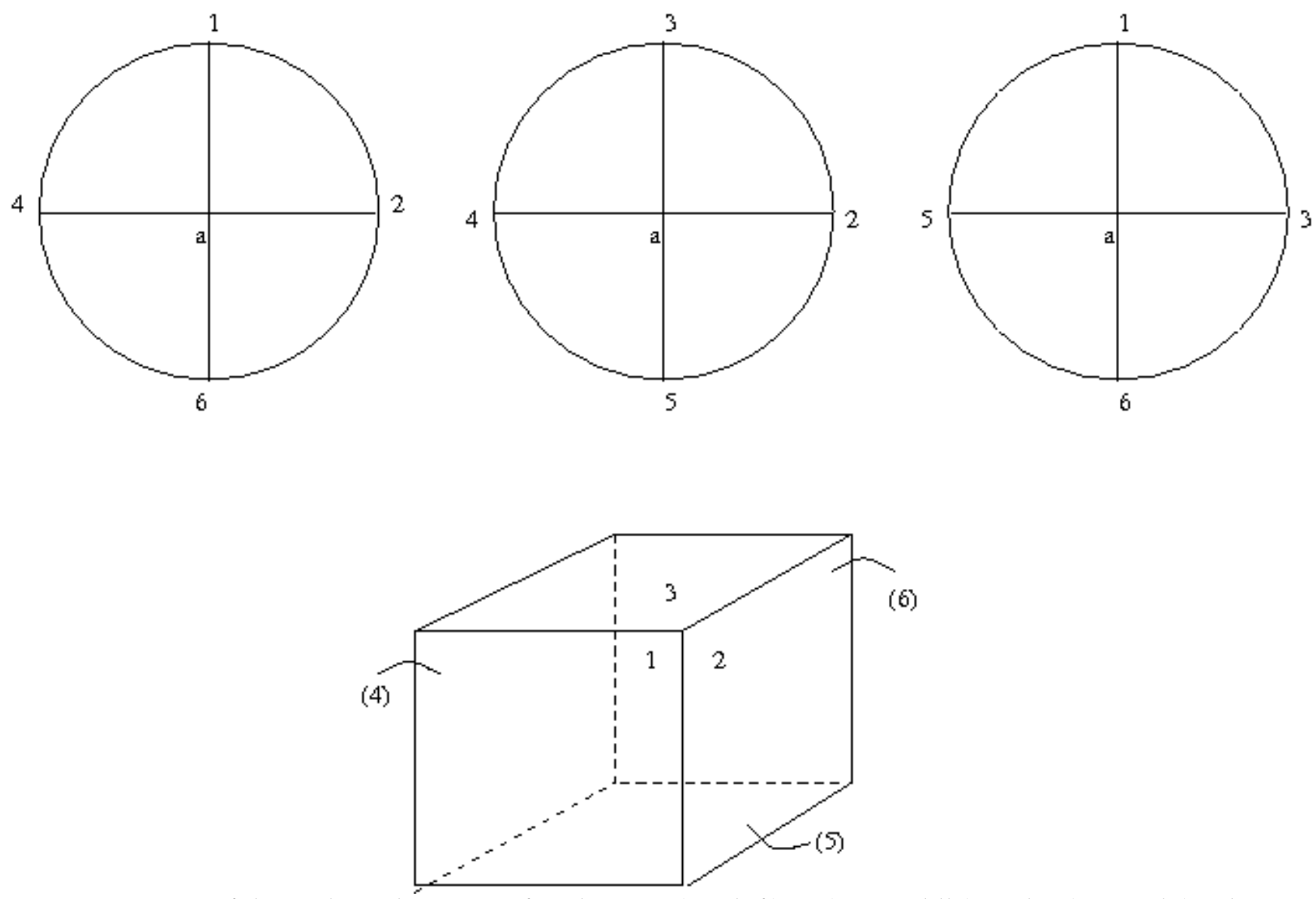

Figure 2: A set of three sheet diagrams, for sheets 1 (top left), 2 (top middle) and 3 (top right). These sheets are dual to a single hex (bottom), with face numbers indicated (back faces are indicated using parentheses). 


\section{ESAIM: PROCEEDINGS}
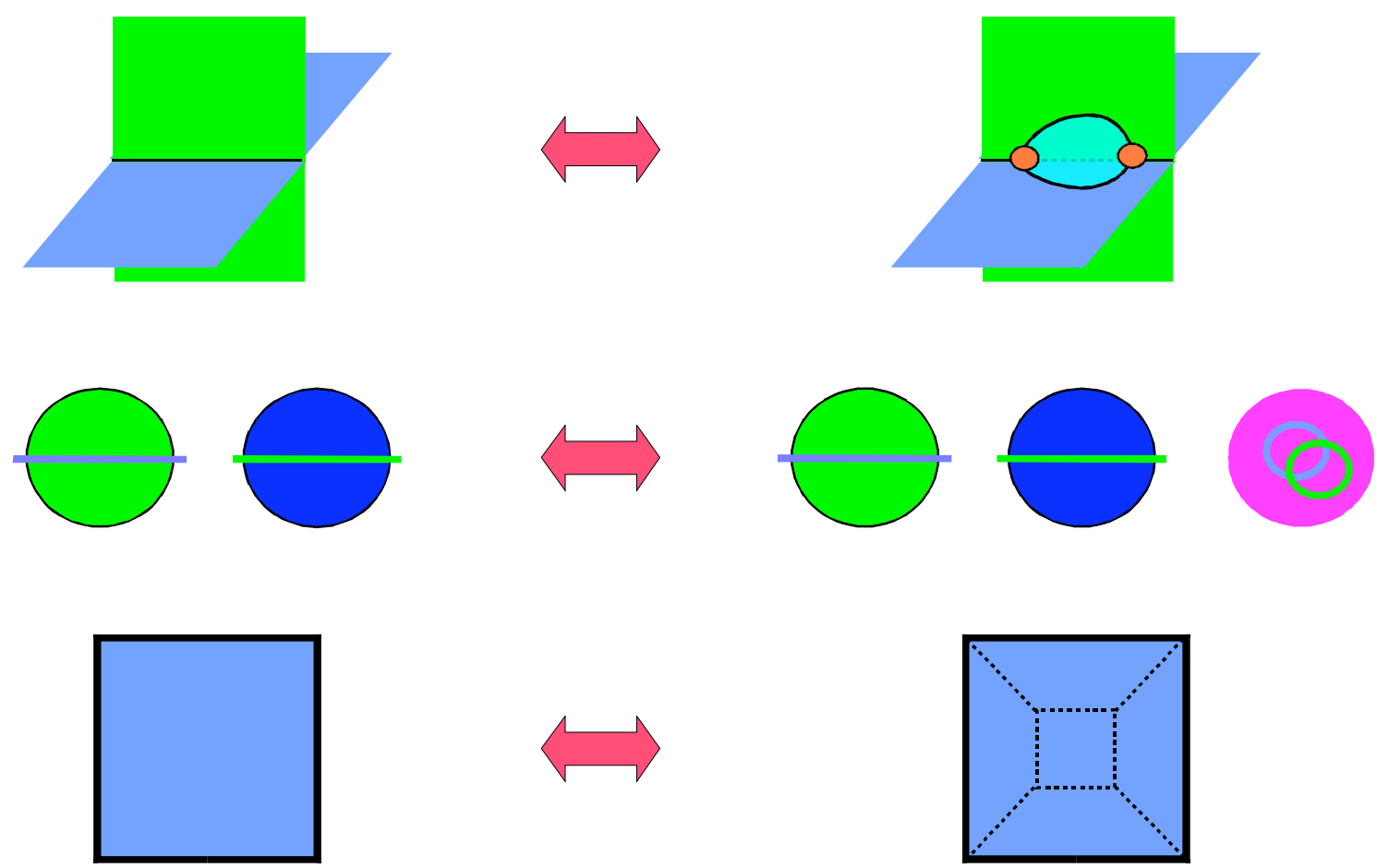

Figure 3: The Atomic Pillow (AP) operation, in the 3D dual (top), sheet diagrams (middle) and primal (bottom). 
ESAIM: PROCEEDINGS, August 2008, Vol. 24, p. 14-33

C. Dobrzynski, P. Frey, Ph. Pebay, Editors
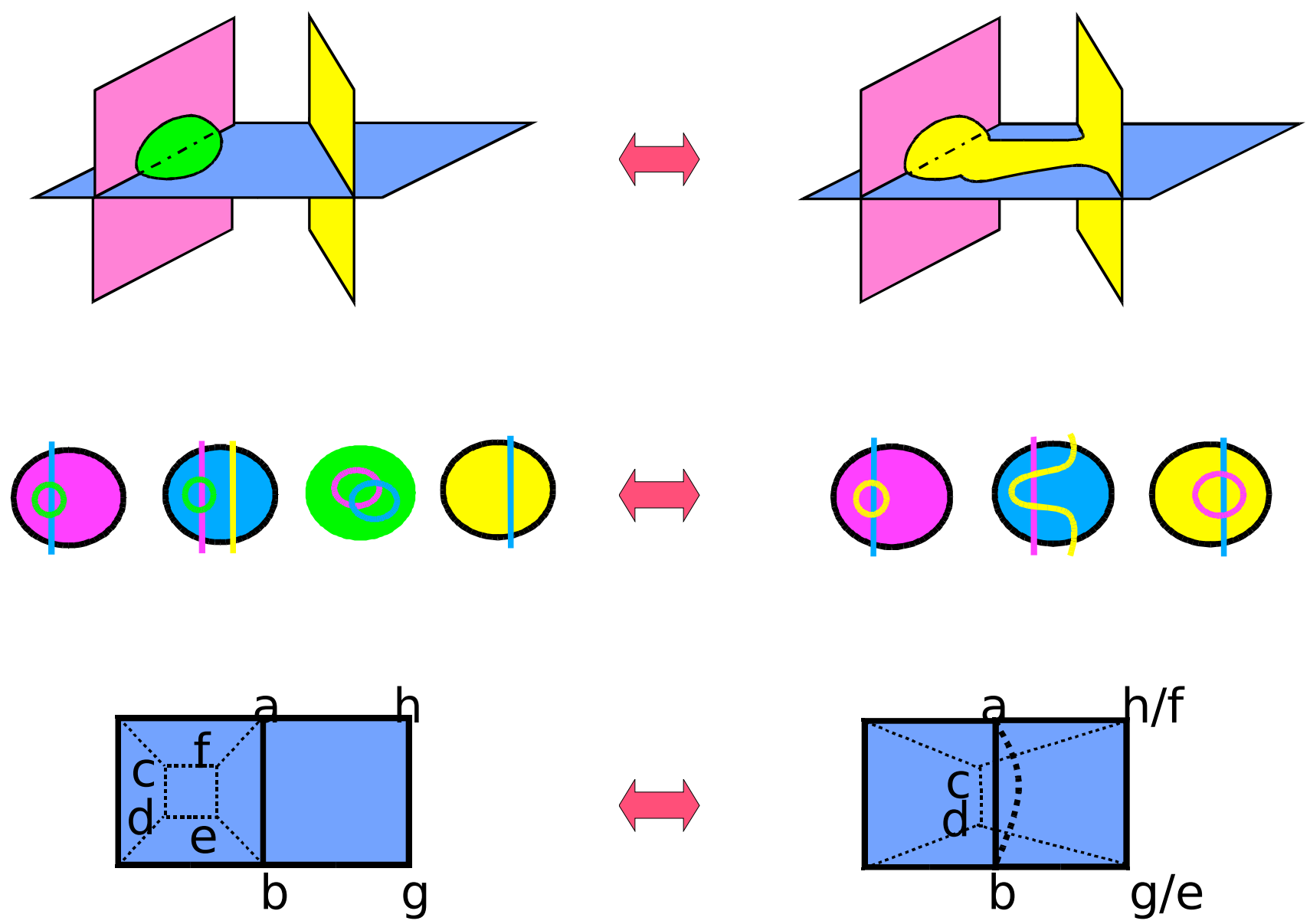

Figure 4: The Open-Collapse (OC) operation, in the 3D dual (top), sheet diagrams (middle) and primal (bottom). 


\section{ESAIM: PROCEEDINGS}
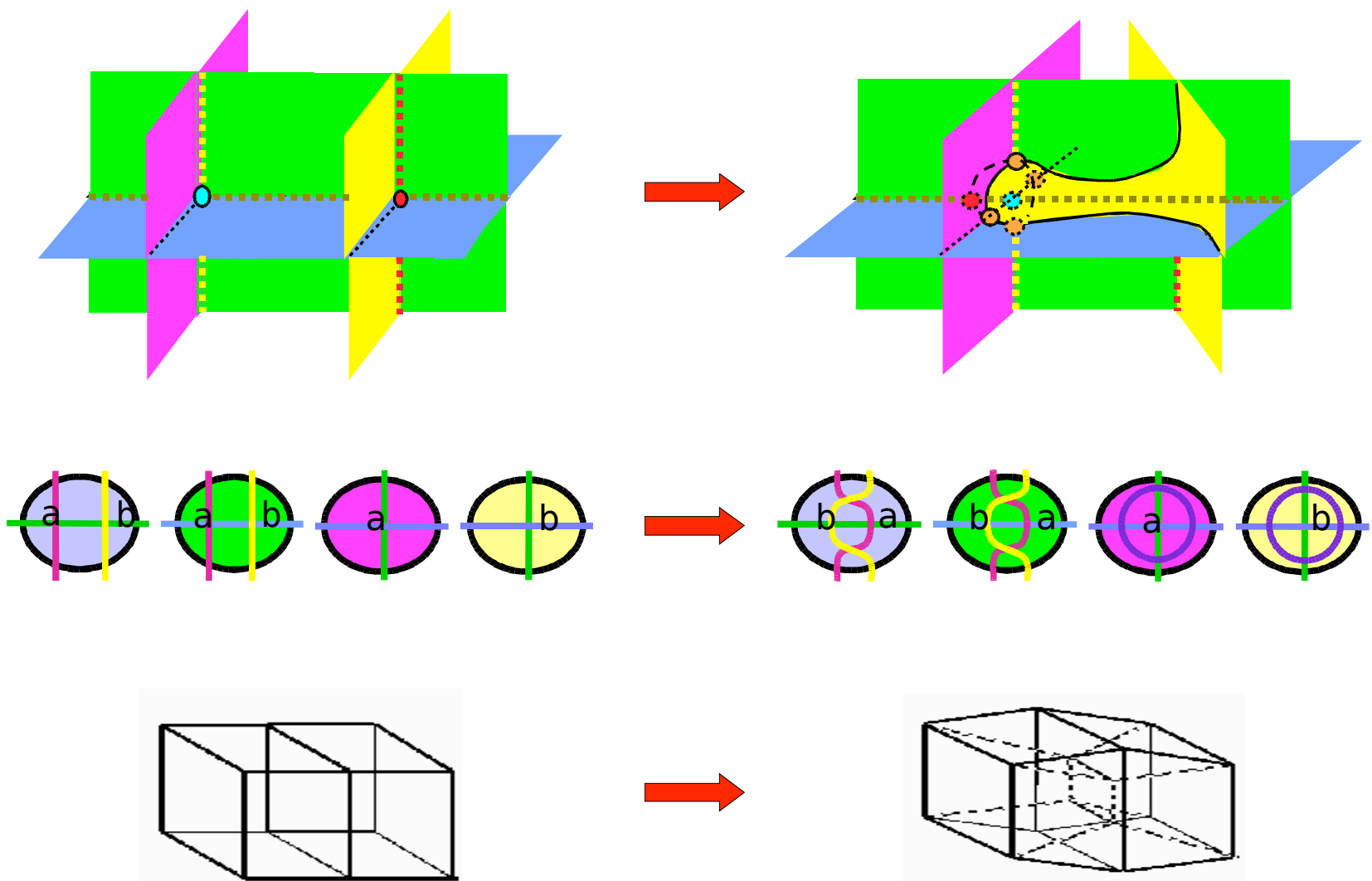

Figure 5: The Face Shrink (FS) operation, in the 3D dual (top), sheet diagrams (middle) and primal (bottom). 
ESAIM: PROCEEDINGS, August 2008, Vol. 24, p. 14-33

C. Dobrzynski, P. Frey, Ph. Pebay, Editors

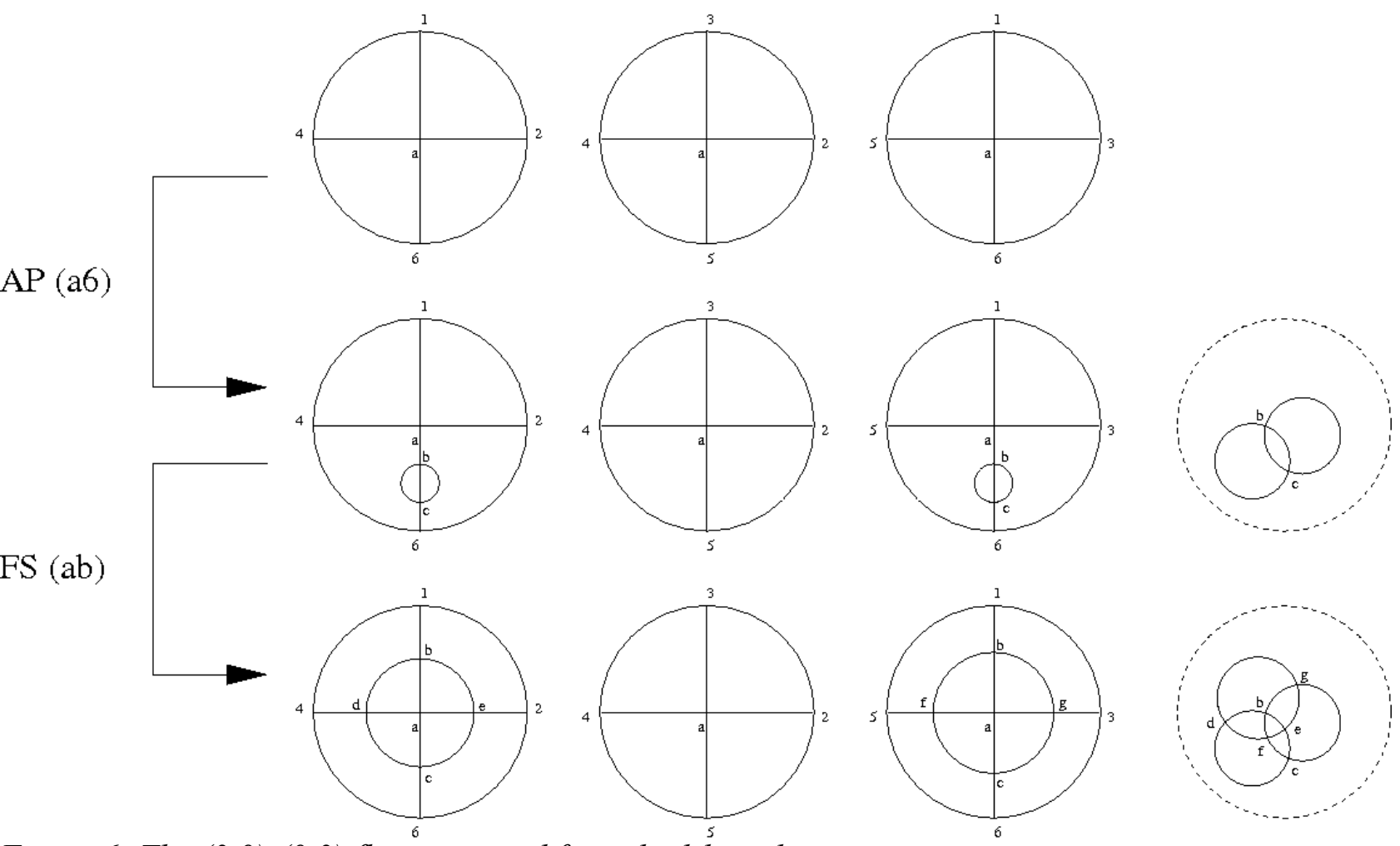

Figure 6: The $(3,0)-(0,3)$ flip composed from dual-based operations. 


\section{ESAIM: PROCEEDINGS}

FS $(a b)$
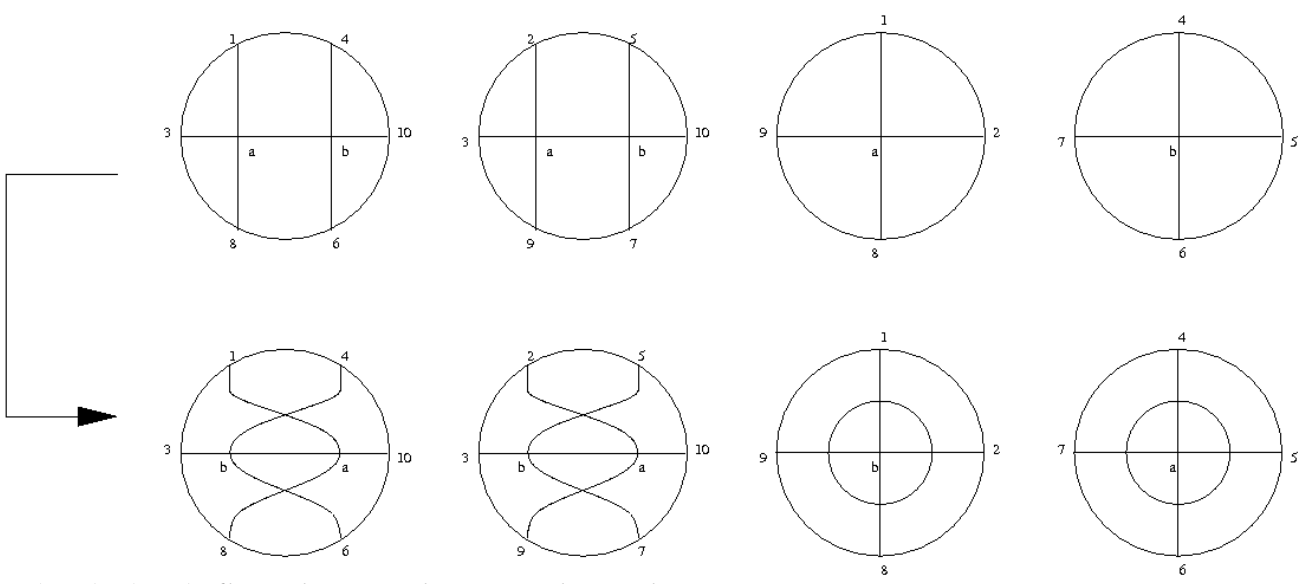

Figure 7: The (2,0)-(0,2) flip, depicted using sheet diagrams.

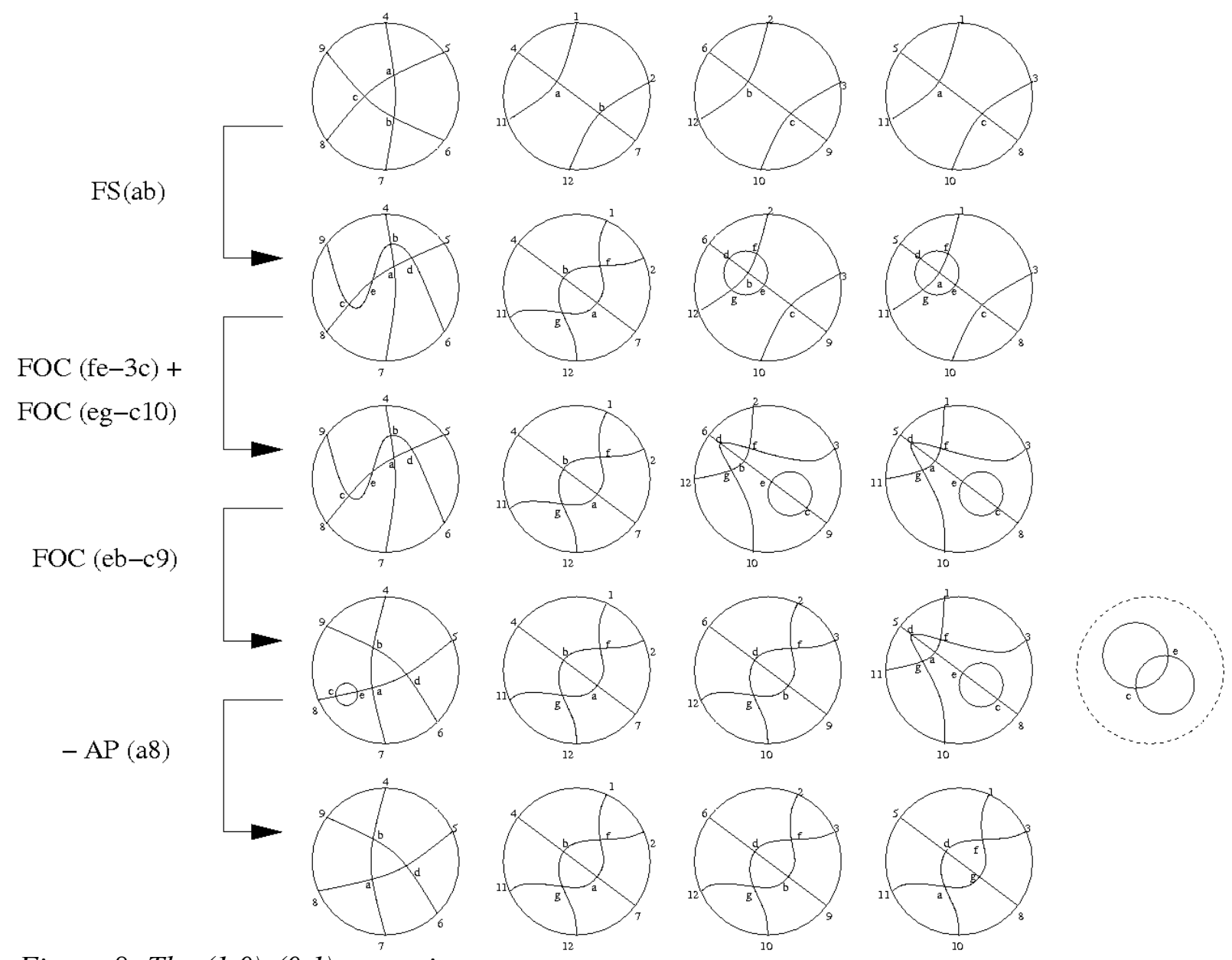

Figure 8: The $(1,0)-(0,1)$ operation. 
ESAIM: PROCEEDINGS, August 2008, Vol. 24, p. 14-33

C. Dobrzynski, P. Frey, Ph. Pebay, Editors

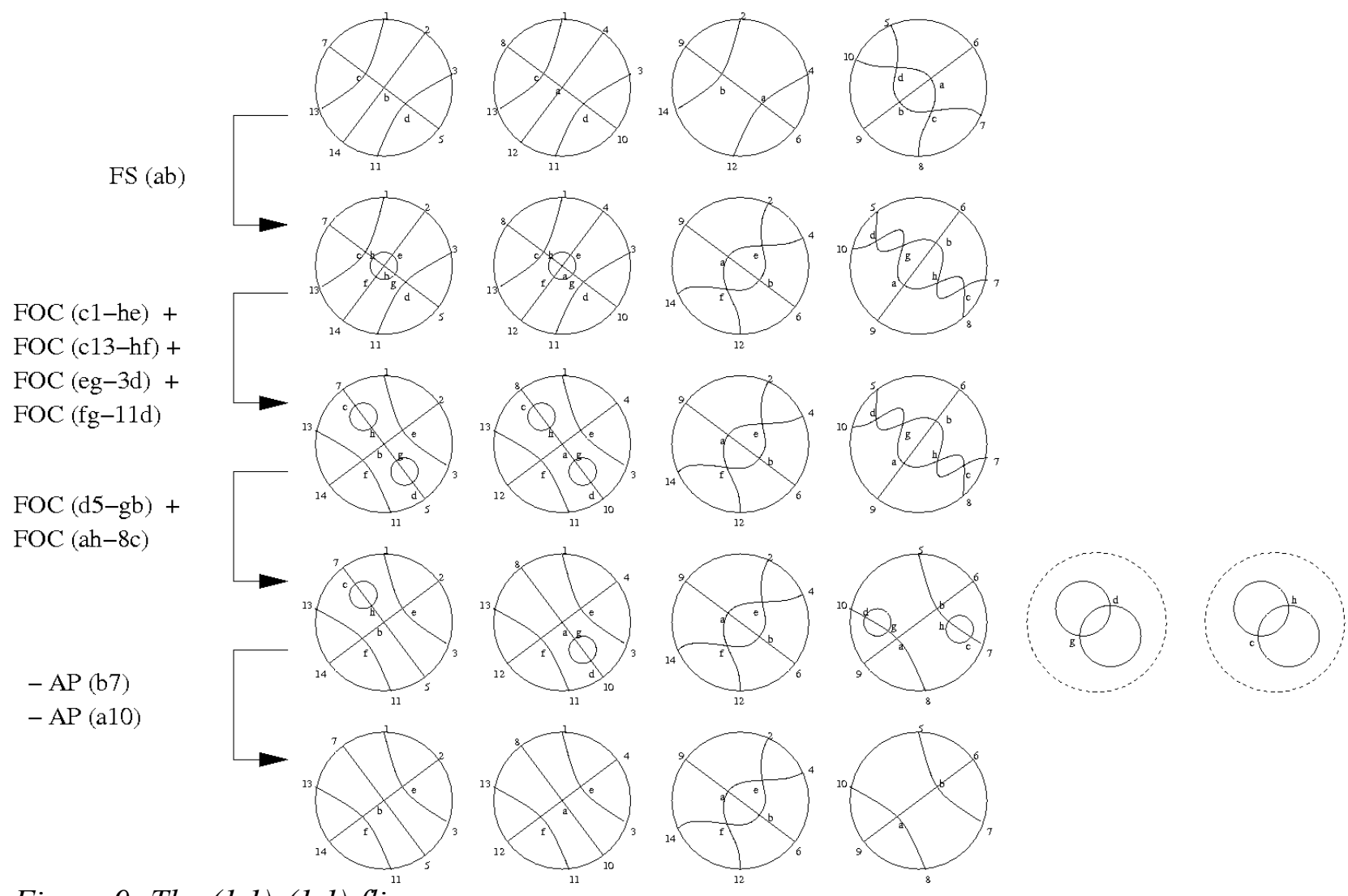

Figure 9: The (1,1)-(1,1) flip. 
ESAIM: PROCEEDINGS

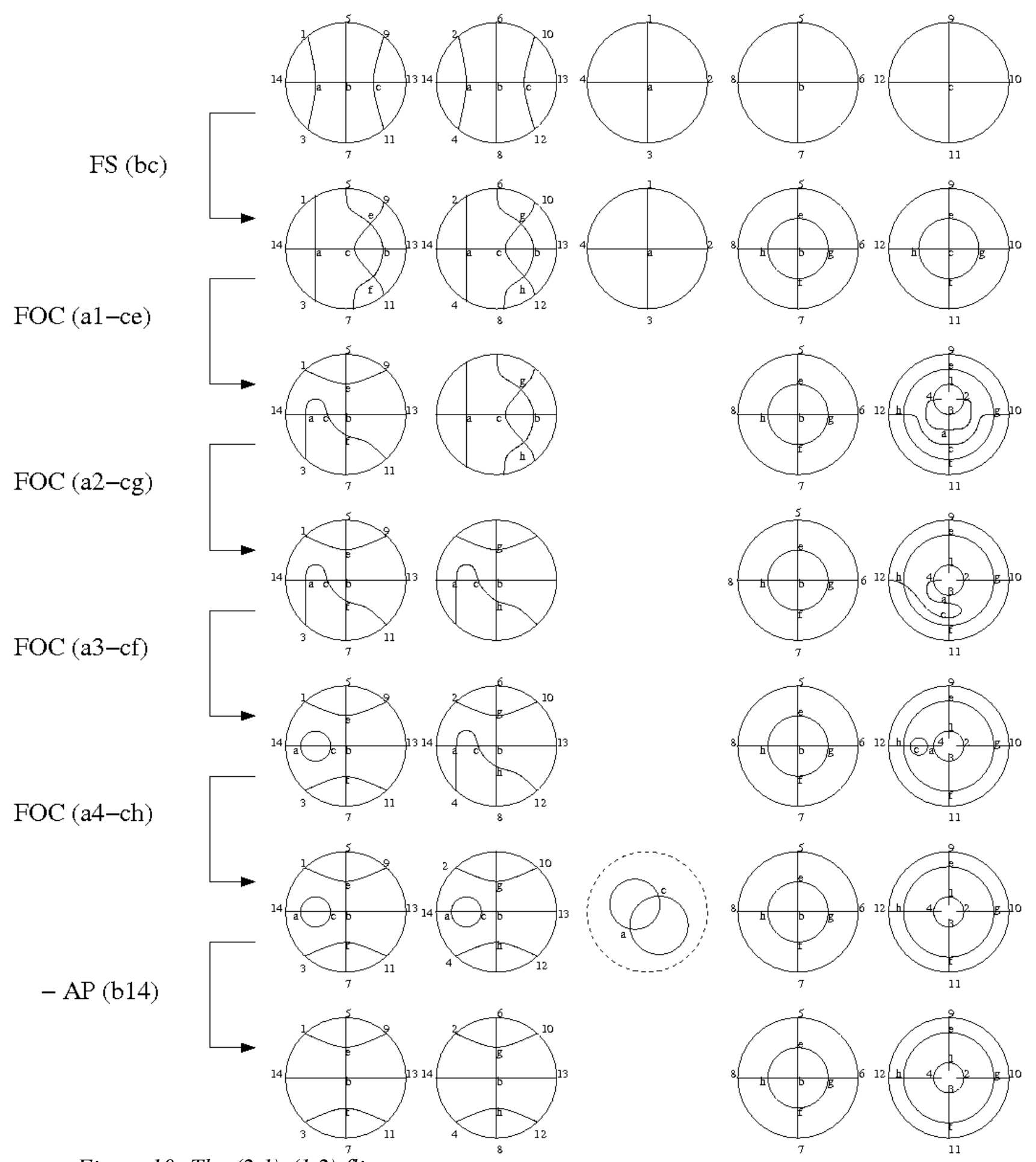

Figure 10: The (2,1)-(1,2) flip. 
ESAIM: PROCEEDINGS, August 2008, Vol. 24, p. 14-33

C. Dobrzynski, P. Frey, Ph. Pebay, Editors

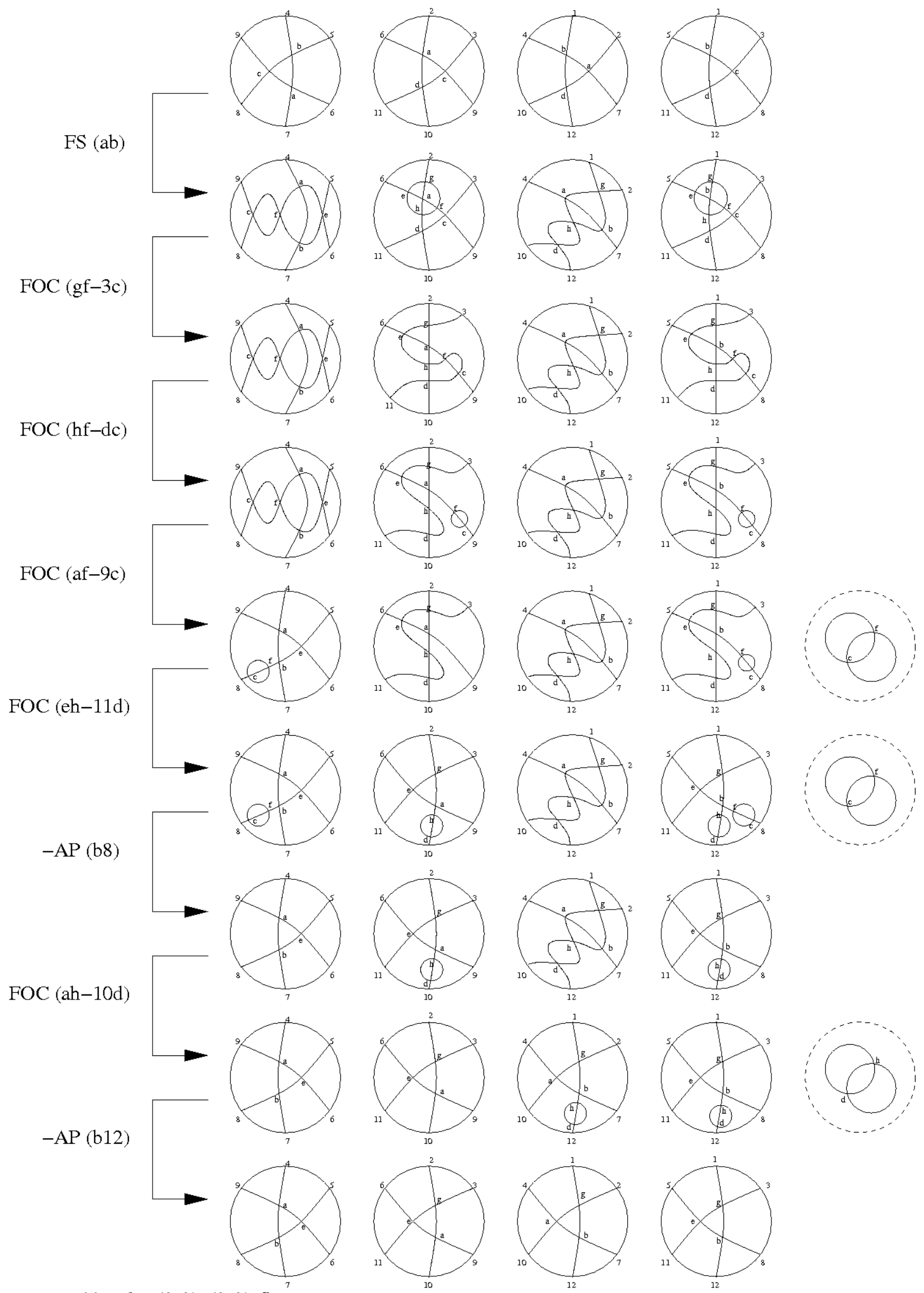

Figure 11: The (0,0)-(0,0) flip. 
ESAIM: PROCEEDINGS
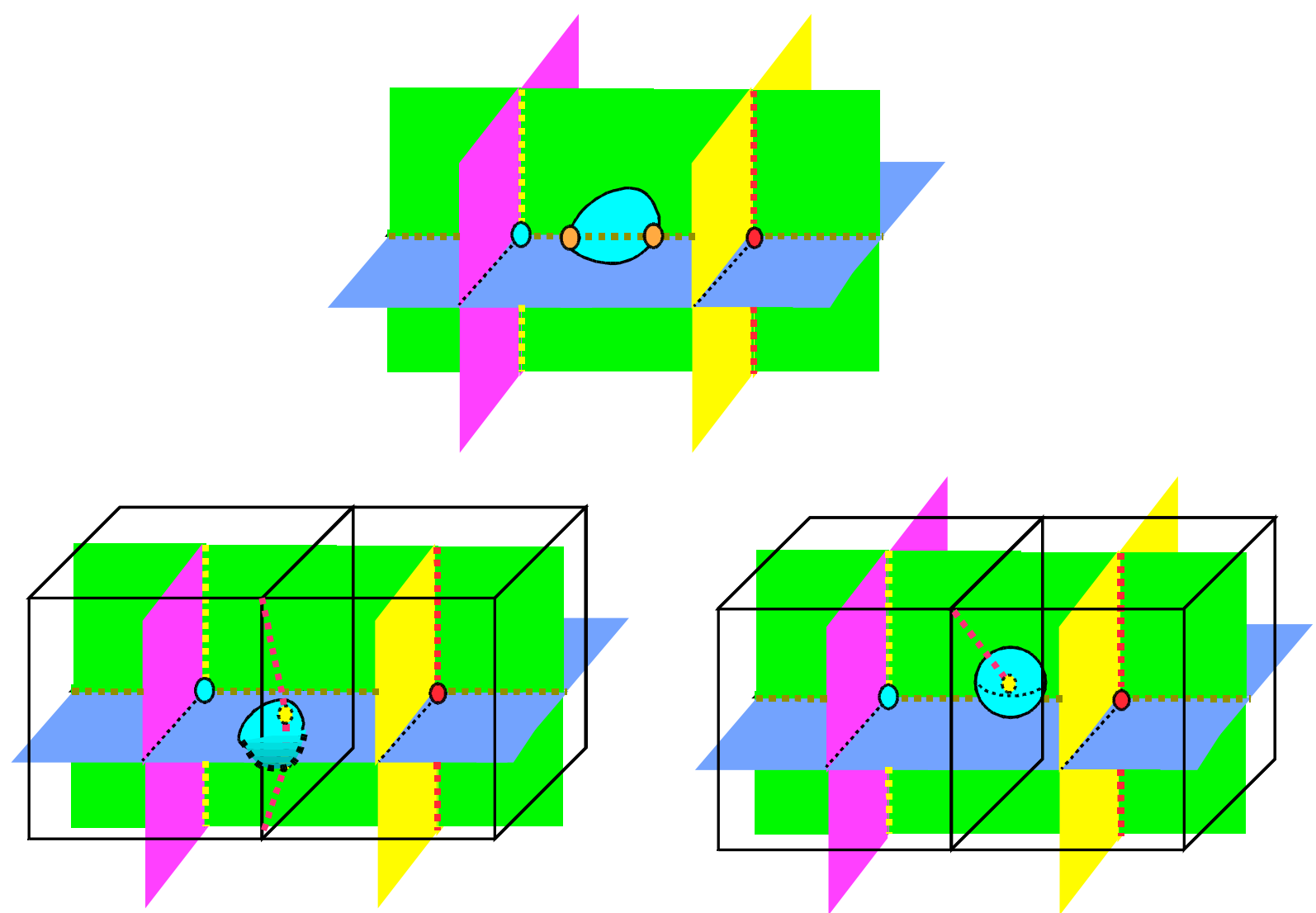

Figure 12: Insertion of a new dual surface which intersects a 1-cell (top), 2-cell (bottom left) or 3-cell (bottom right). 
ESAIM: PROCEEDINGS, August 2008, Vol. 24, p. 14-33

C. Dobrzynski, P. Frey, Ph. Pebay, Editors

a)
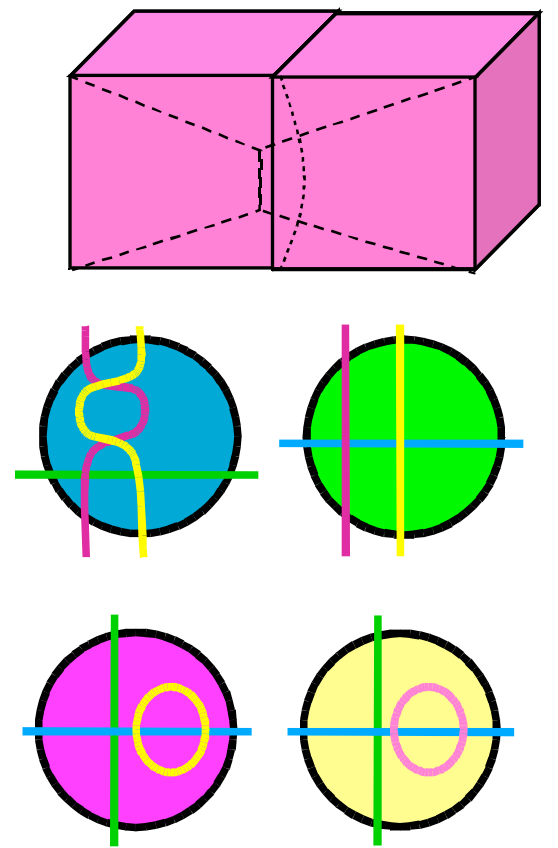

c)
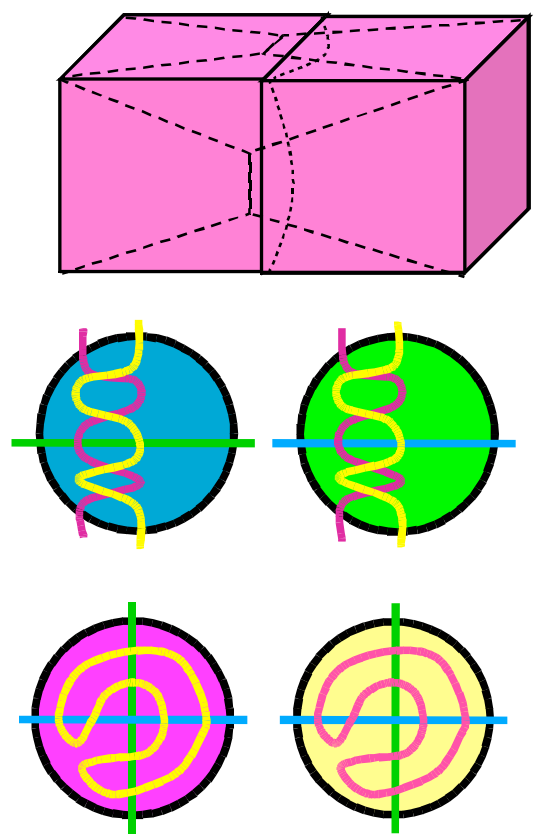

e)
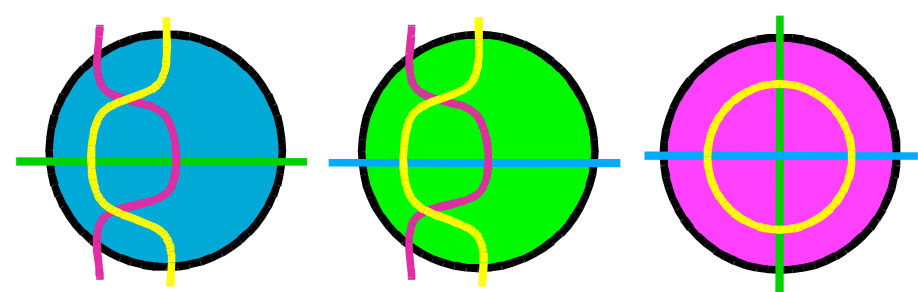

d)
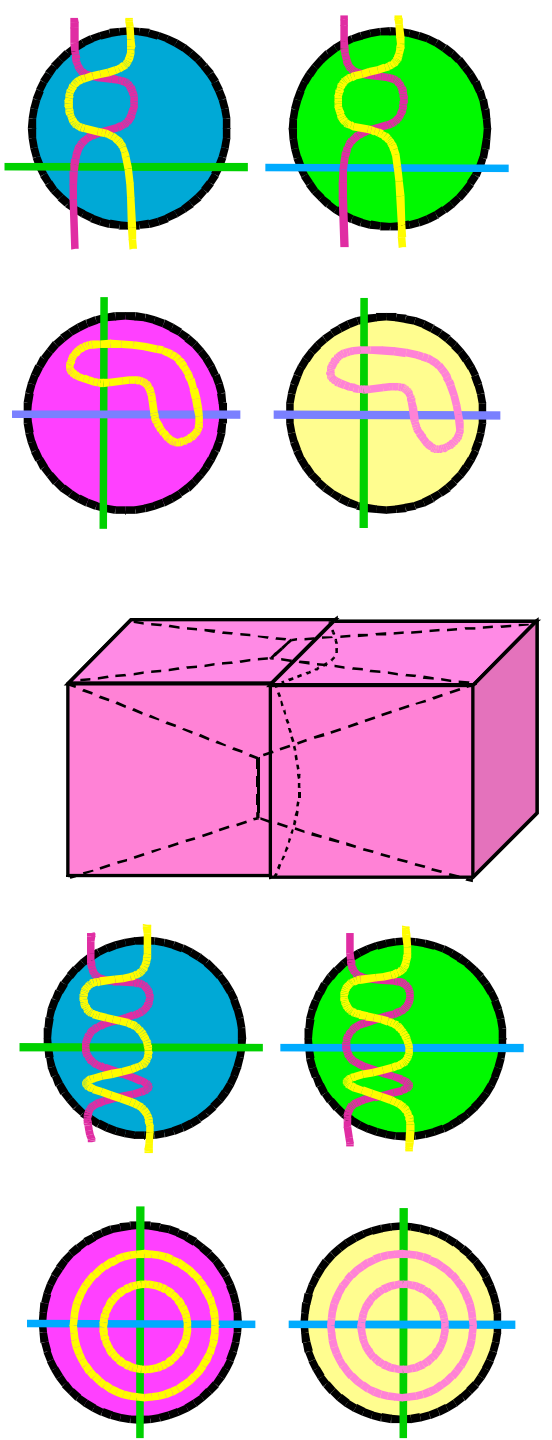

Figure 13: Relating FS to AP and OC. a) Application of AP, OC; b) another AP, OC; c) another $A P, O C$; d) application of $O C$ to join "ring" of hexes; e) application of reverse FS. 
ESAIM: PROCEEDINGS
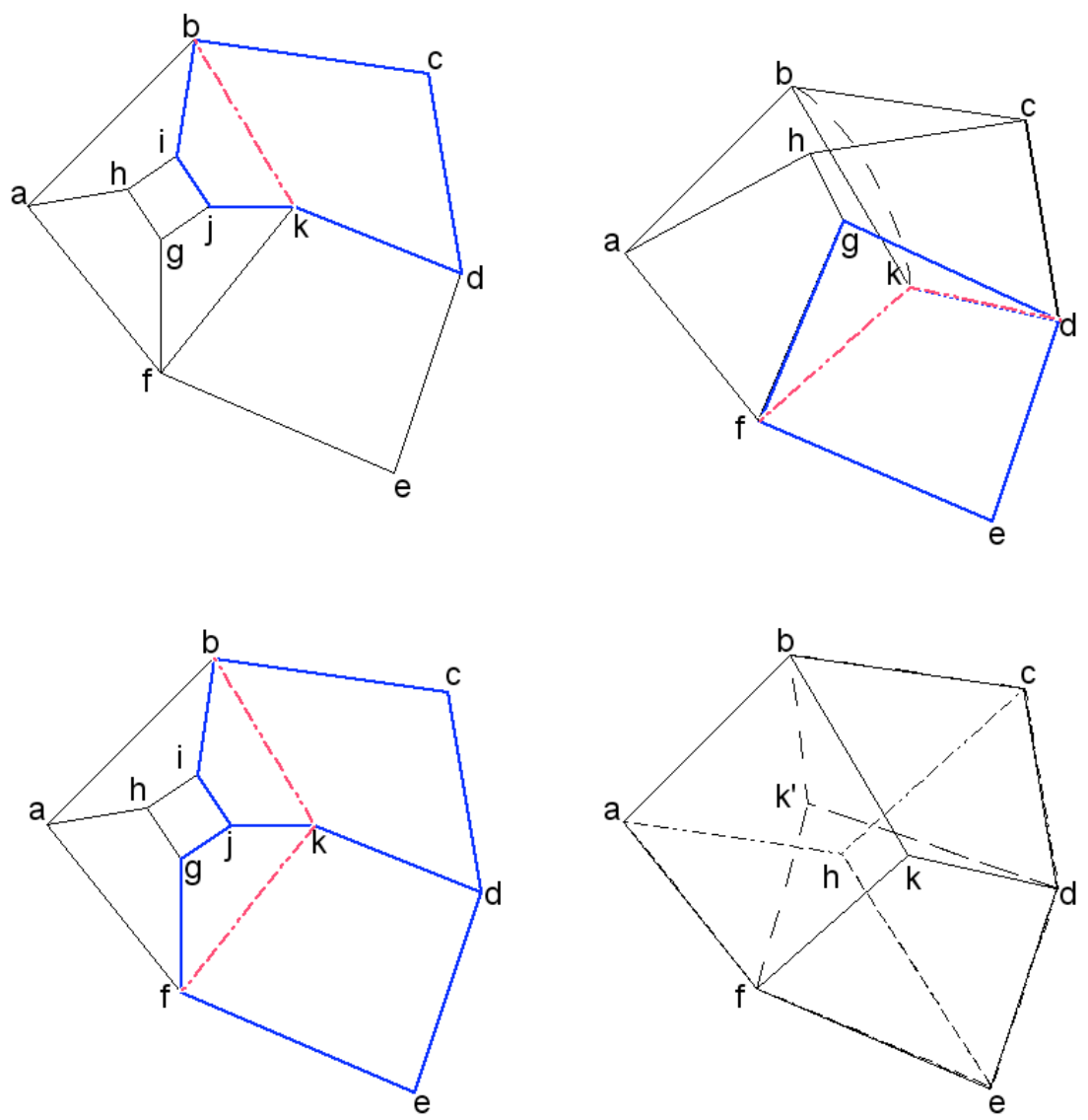

Figure 14: Different forms of the Open/Collapse operation. 